\title{
EXPERIÊNCIA DO USUÁRIO COM APLICATIVO DE SAÚDE: UM ESTUDO PILOTO NA REDE PÚBLICA DA REGIÃO NORTE DO RIO GRANDE DO SUL
}

\author{
USER EXPERIENCE IN HEALTH APPS: A PILOT STUDY IN THE PUBLIC HEALTH NETWORK OF \\ THE NORTHERN REGION OF RIO GRANDE DO SUL
}

\section{EXPERIENCIA DEL USUARIO EN APPS DE SALUD: UN ESTUDIO PILOTO EN LA RED DE SALUD PÚBLICA DE LA REGIÓN NORTE DE RIO GRANDE DO SUL}

\author{
Simiane Salete Volpia, Raquel Debona, Daiana Biduskia, Danieli Tefilia, Ericles \\ Andrei Bellei*a, Ana Luisa Sant'Anna Alvesa, Ana Carolina Bertoletti De Marchía \\ Universidade de Passo Fundoa \\ *E-mail: 168729@upf.br
}

\begin{abstract}
RESUMO
Os aplicativos de saúde envolvem um número crescente da população, motivados pelo interesse nos avanços tecnológicos. Dessa forma, este estudo analisou a experiência e a aceitação do usuário na utilização de um aplicativo para o monitoramento da hipertensão. Participaram do estudo 11 usuários portadores de hipertensão, que utilizaram o aplicativo por três meses. Após o uso, os participantes responderam questionários de avaliação da experiência (SUPR-Qm), de avaliação da tecnologia (TAM) e o sociodemográfico. Para a abordagem qualitativa, investigou-se aspectos do aplicativo considerados importantes para os usuários. O estudo possibilitou identificar as experiências, percepções e necessidades dos usuários ao utilizar um aplicativo para o acompanhamento da hipertensão. Entretanto, sugere-se a realização de estudos futuros para analisar a experiência e a aceitação cumulativa ao longo do tempo, buscando-se comparar os resultados antes e depois da interação com o aplicativo.
\end{abstract}

Palavras-chave: Serviço de saúde comunitária; hipertensão; tecnologia; informática em saúde pública; aplicações da informática médica.

\section{ABSTRACT}

Health applications can involve a lot of people, motivated by the interest in technological advances. Thus, this study analyzed user experience and acceptance when using an application for monitoring hypertension. Participated in the study eleven hypertensive users who used the application for three months. After the use, participants answered the following questionnaires: (SUPR-Qm), technology assessment (TAM) and the sociodemographic. For the qualitative approach, we investigated aspects of the application considered important to users. The study's results identified users' experiences, perceptions and needs while interacting with an application to monitor hypertension. However, future studies are suggested to analyze experience and cumulative acceptance over time, seeking to compare results before and after interaction with the application.

Keyword: Community health service; hypertension; technology; public health informatics; medical informatics applications.

\section{RESUMEN}

Las aplicaciones móviles de salud son cada vez más utilizadas por las personas a causa del interés en los avances tecnológicos. Por lo tanto, este estudio analizó la experiencia del usuario y su aceptación al usar una aplicación para monitorear la hipertensión. Once usuarios hipertensos que utilizaron la aplicación durante tres meses participaron en el estudio. Después del uso, los participantes respondieron cuestionarios (SUPR-Qm), evaluación de tecnología 


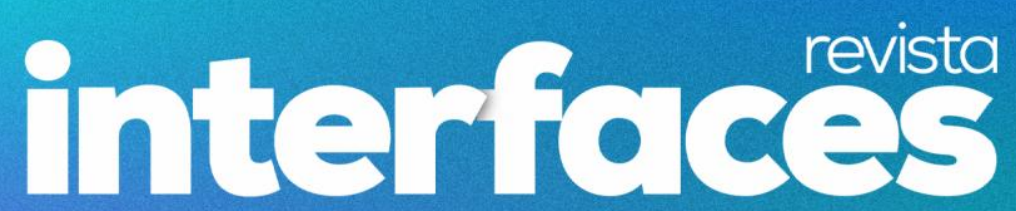

\section{Artigos \\ Originais}

V.10 N.1 (2022) ISSN: 2317-434X

(TAM) y cuestionarios sociodemográficos. Para el enfoque cualitativo, fueron investigados aspectos de la aplicación considerados importantes para los usuarios. El estudio permitió identificar las experiencias, percepciones y necesidades de los usuarios mediante el uso de una aplicación para controlar la hipertensión. Sin embargo, se sugieren estudios futuros para analizar la experiencia y la aceptación acumulativa a lo largo del tiempo, buscando comparar los resultados antes y después de la interacción con la aplicación móvil.

Palabras clave: servicio comunitario de salud, hipertensión, tecnología, informática de salud pública, aplicaciones de informática médica 


\section{INTRODUÇÃO}

A hipertensão acomete diversas faixas etárias e a baixa adesão ao tratamento contribui para o controle inadequado da pressão arterial, podendo elevar o risco de crises hipertensivas, bem como a evolução para a internação hospitalar. A hipertensão é considerada uma doença crônica não transmissível, muitas vezes silenciosa, que aumenta o risco de outras doenças e pode levar a mortalidade precoce, acarretando impacto econômico, social e de saúde para o indivíduo e sua família (FREITAS et al., 2018; SILVA et al., 2017). Atualmente, um terço das pessoas com hipertensão não é diagnosticada e, dentre as diagnosticadas, metade não adere ao tratamento medicamentoso e nem segue as recomendações dos profissionais da saúde (FREITAS et al., 2018; KITT et al., 2019).

A adoção de medidas saudáveis associada ao uso de aplicativos tem inovado no controle dos níveis pressórios e na redução de peso dos pacientes préhipertensão e com hipertensão (HARICHARAN et al., 2017; TORO-RAMOS et al., 2017). Entretanto, há necessidade de investimentos de métodos que possam auxiliar na modificação de estilo de vida e no monitoramento da hipertensão.

Os aplicativos focados na área da saúde vêm atraindo um número crescente de usuários, motivados pelo advento e difusão dos dispositivos móveis. Esses aplicativos podem auxiliar na mudança de comportamento, mantendo o usuário engajado durante o uso e, consequentemente, aumentando a adesão ao tratamento por meio do acompanhamento da hipertensão. Esses aplicativos são ferramentas que agregam as orientações e monitoram à saúde desde as atividades diárias (VO; AUROY; SARRADON-ECK, 2019). Eles representam melhorias no envolvimento dos profissionais de saúde com maior aceitação e conforto (DAVIS et al., 2015; SILVA et al., 2017).

Os aplicativos também facilitam a interação entre os profissionais de saúde e os pacientes antes e depois das consultas8. Dessa forma, o paciente também pode compreender as alternativas de cuidado mediante soluções de acompanhamento por meio da tecnologia, o que pode acarretar em mais confiança para o usuário e gerar menor resistência ao registrar dados em um sistema (DOU et al., 2017; MÁRQUEZ CONTRERAS et al., 2019; SUN et al., 2017).

Neste sentido, é necessário considerar as impressões dos usuários perante o uso de aplicativos para saúde. A experiência do usuário aborda as impressões, expectativas, e consequências emocionais e comportamentais de cada indivíduo perante o uso de um produto (BIDUSKI et al., 2020; CAVALCANTE; RIVERO; CONTE, 2015). O estímulo do usuário para o uso do aplicativo contribui para o autogerenciamento e autocuidado da doença. Diante deste contexto, o objetivo deste estudo foi analisar a experiência e aceitação da utilização de um aplicativo de saúde em pacientes hipertensos atendidos na rede pública de saúde.

\section{MÉTODO}

Trata-se de um estudo piloto de abordagem mista, que combina elementos de pesquisa quantitativoqualitativo (JOHNSON; ONWUEGBUZIE; TURNER, 2007). O estudo faz parte de um projeto guarda-chuva financiado pelo Ministério da Saúde e Conselho Nacional de Desenvolvimento Científico e Tecnológico (DE MARCHI et al., 2020). Os objetivos da pesquisa foram esclarecidos e os participantes assinaram o Termo de Consentimento Livre e Esclarecido.

A coleta dos dados ocorreu em local reservado de uma Unidade Básica de Saúde por entrevistadores treinados. $\mathrm{O}$ aplicativo utilizado na intervenção é denominado e-Lifestyle (CECHETTI et al., 2019). O objetivo é possibilitar aos pacientes o controle, gerenciamento e monitoramento da hipertensão. O aplicativo é parte integrante de uma plataforma para comunicação entre o profissional da saúde e o paciente hipertenso. O paciente pode registrar e acompanhar informações sobre sua saúde, como pressão arterial, sono, humor, peso, entre outros.

Os participantes deste estudo foram pacientes hipertensos, adultos e idosos, que utilizaram o aplicativo por um período de três meses. Foram incluídos os participantes com cognitivo preservado, comprovado por meio do Mini Exame do Estado Mental, com condições de verificar a pressão em farmácias, hospitais, UBS ou CAIS, que possuíam um smartphone com SO Android versão igual ou superior a 5.0; com afinidade no uso de aplicativos; e com Internet em casa (Wi-fi) ou no celular (Dados Móveis). Foram excluídos os participantes que não registraram as informações no aplicativo por mais de dez dias.

$\mathrm{O}$ instrumento inicial da avaliação com os participantes possibilitou a abordagem das características sociodemográficas (idade, gênero, cor de pele, estado civil, renda mensal, escolaridade e profissão). Além disso, foram utilizados dois questionários para avaliação da experiência com o aplicativo, juntamente com uma pergunta aberta: o questionário SUPR-Qm, composto por 12 questões que abordam temas relacionados à facilidade, preferência, atração, intenção de uso, e expectativas do usuário (SAURO; ZAROLIA, 2017); e o questionário de 


\section{V.10 N.1 (2022) ISSN: $2317-434 \mathrm{X}$}

aceitação da tecnologia (TAM), composto por 15 itens, que avalia as utilidades percebidas, facilidades, atitudes e resultados demonstrados no momento do uso (DAVIS; BAGOZZI; WARSHAW, 1989). No questionário de experiência e de aceitação, optou-se pela escala de Likert, com cinco alternativas de respostas, sendo cinco o maior valor: concordo totalmente (5), concordo (4), neutro (3), discordo (2) e discordo totalmente (1). Por fim, aplicou-se uma pergunta aberta de resposta livre: $\mathrm{O}$ que você mais gostou no aplicativo e por quê?

Os dados quantitativos foram digitados e analisados em software de estatística, sendo conduzidas análises descritivas. Para a análise da pergunta de resposta livre, foram descritas as respostas e os participantes identificados de P1 à P11.

\section{RESULTADOS}

Foram avaliados 11 pacientes com média de idade de 52,27 anos ( $D P=15,66$; mínima=20; máxima=74), sendo que $18,2 \%$ eram do sexo masculino e $81,8 \%$ do sexo feminino. Do total de participantes, $72,7 \%$ eram de cor da pele branca, 63,6\% viviam com companheiro, 54,6\% tinham pelo menos o ensino médio completo e $72,7 \%$ recebiam até 2 salários mínimos (Tabela 1).

Tabela 1. Descrição das características demográficas e socioeconômicas de usuários de unidades básicas de saúde do município de Passo Fundo, RS, 2019 (n=11).

\begin{tabular}{llcc}
\hline Variável & Categorias & $\mathbf{N}$ & \% \\
\hline Gênero & Masculino & 2 & 18,2 \\
& Feminino & 9 & 81,8 \\
\hline Cor da pele & Branca & 8 & 72,7 \\
& Não branca & 3 & 27,3 \\
\hline Estado civil & Sem companheiro & 4 & 36,4 \\
& Com companheiro & 7 & 63,6 \\
\hline Escolaridade & Ensino fundamental incompleto & 27,3 \\
& Ensino fundamental completo & 3 & 18,2 \\
& Ensino médio completo & 2 & 45,5 \\
& Pós-Graduação & 5 & 9,1 \\
\hline Renda & Até 2 salários mínimos & 1 & 72,7 \\
& 2 à 4 salários mínimos & 8 & 27,3 \\
\hline
\end{tabular}

Para o questionário de experiência, SUPR-Qm, foram realizadas medidas de tendência central e dispersão para cada questão e para o conjunto de questões. A média do SUPR-Qm foi de 3,8 ( $\mathrm{DP}=0,75)$, ou seja, entre concordo (4) e neutro (3). Na análise por questões, apresentaram menor concordância as questões 5, 8, 10 e 11 , as demais apresentaram variação entre concordo totalmente (5) e concordo (4) (Figura 1). Quanto a frequência absoluta e relativa simples de respostas do SUPR-Qm, as respostas variaram de discordo a concordo totalmente. Nas questões 3, 7, 9 e 12, mais de $50 \%$ dos participantes concordaram totalmente. As questões 1, 2, 3 e 6 não apresentaram nenhum grau de discordância. Os resultados das medidas de tendência central e dispersão do questionário de aceitação da tecnologia (TAM) estão apresentados na Figura 2. A média geral do questionário foi de 4,2 ( $\mathrm{DP}=0,58)$, variando de concordo totalmente a concordo. As menores médias foram observadas nas questões 7 e 14, as demais questões tiveram médias superiores a 4 . 


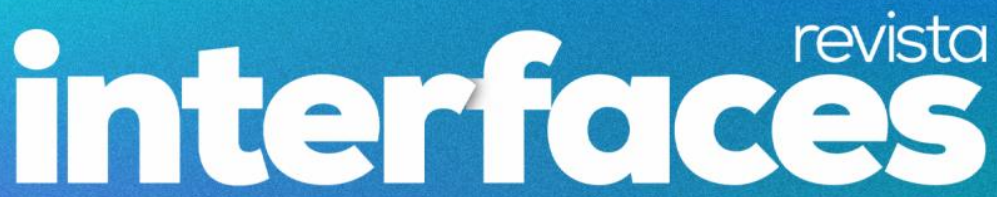

Artigos

Originais

V.10 N.1 (2022) ISSN: $2317-434 \mathrm{X}$

Figura 1. Descrição das medidas de tendência central e dispersão de cada questão do SUPR-Qm, 2019.

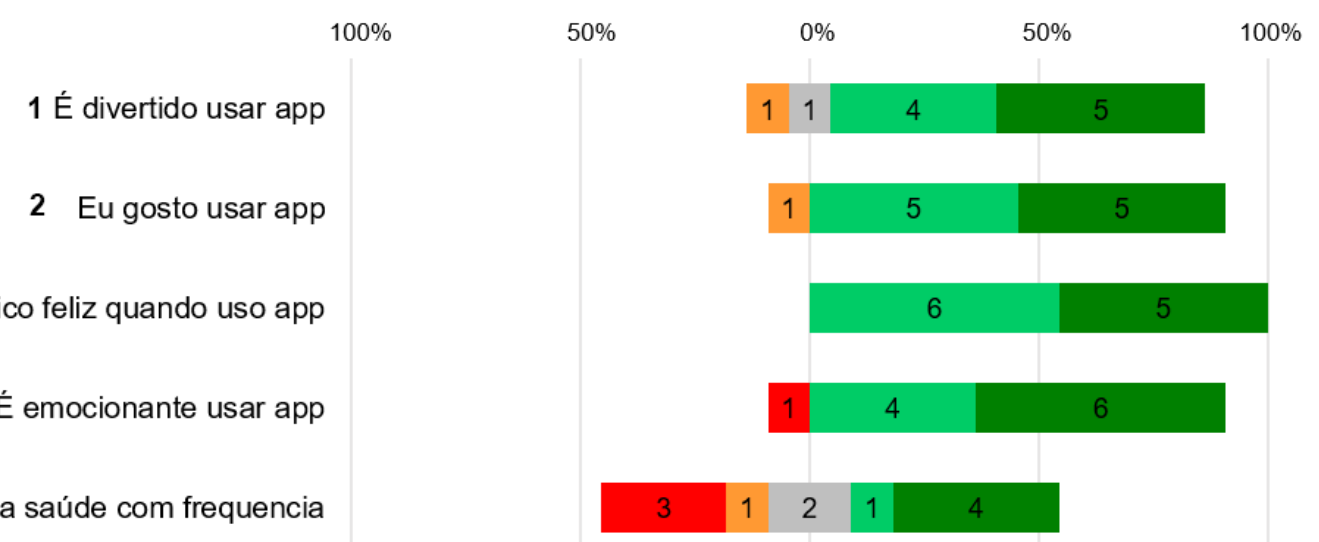

5 Eu uso app para a saúde com frequencia

6 Eu confio em manter minhas informações pessoais no app

7 É facil navegar dentro app

8 Eu acho o app atraente

9 O app é simples e claro

10 Eu gosto de descobrir novos recursos no app

11 Eu falo sobre as coisas do app com meus amigos

12 Eu recomendaria o appp para outras pessoas

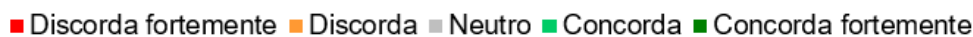


V.10 N.1 (2022) ISSN: $2317-434 \mathrm{X}$

Figura 2. Descrição das medidas de tendência central e dispersão de cada questão do TAM, 2019.

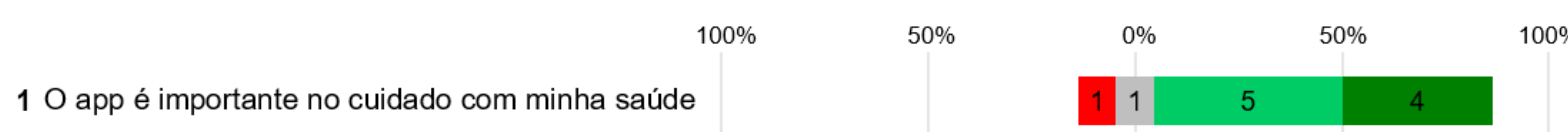

2 O app é útil para gestâo da minha saúde

3 O app torna o cuidado com minha saúde mais interessante

4 O app ajuda manter o histórico e a evolução da minha saúde

5 O app aumenta o cuidado com minha saúde

6 O app motiva meu envolvimento no controle da minha saúde

7 A minha interaçã ocom app é clara e compreensível

8 A interação não exige um elevado esforço mental

9 O app é fácil d usar

10 É fácil aprender a usar o app

11 Houve treinamento para usa o app

12 O pesquisador tem bom nivel de conhecimento sobre app me ajudou a entendê-lo

13 Seria melhor usar o app em vez de usar o método de acompnhamento que utilizo atualmente

14 Os resultados da utilização do app são inquestionáveis

15 Continuarei a usar o app no cuidado com minha saúde

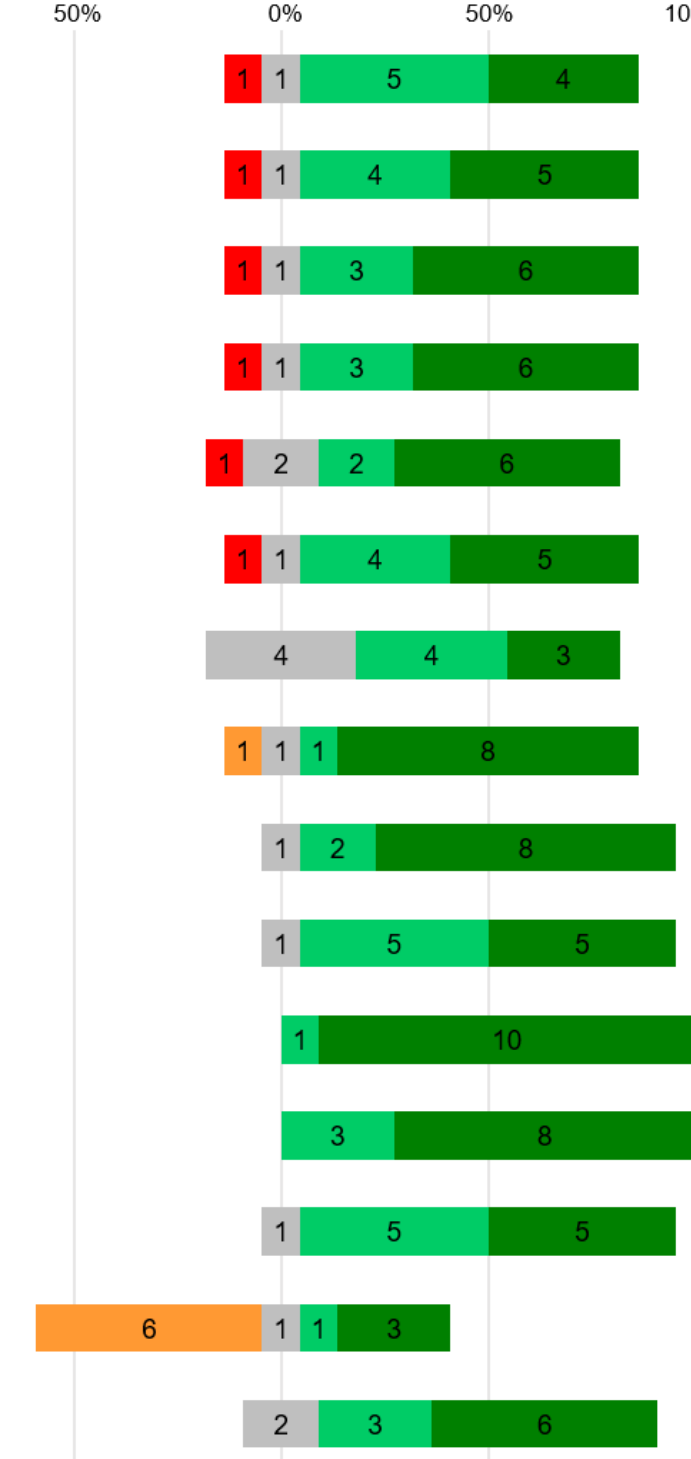

$\square$ Discorda fortemente $=$ Discorda $₫$ Neutro $\backsim$ Concorda $\backsim$ Concorda fortemente

de sensações agradáveis e preferem experiências que possam melhorar as condições de saúde no cotidiano (LU et al., 2018). Após o uso, os usuários afirmaram que o aplicativo era simples e fácil de utilizar. Uma experiência gratificante faz com que os usuários continuem usando o aplicativo por um longo período de tempo e o indiquem para outras pessoas (BIDUSKI et al., 2020). No geral, a experiência foi considerada inovadora, com base nas orientações fornecidas de forma explicativa, o que acarretou em mais segurança e confiança para os usuários no momento de inserir as informações e o explorar 
aplicativo(ANDERSEN et al., 2017; BOUDREAUX et al., 2014; DOU et al., 2017).

Em contrapartida, houve menor concordância na questão 5 , onde foi abordada a frequência de uso do aplicativo. A falta de integração da tecnologia na vida diária, a pouca comunicação e o interesse reduzido de alguns participantes podem ser oriundas de limitações e problemas técnicos, assim como no estudo de (WOODS et al., 2019). Com base nessas colocações, foram reforçadas orientações de esclarecimento e monitoramento, porém, mesmo assim, esses usuários permaneceram desinteressados. Geralmente, usuários tomam decisões sobre a aceitação do aplicativo no início do período de utilização. Portanto, muitas vezes não se sentem motivados para solucionar problemas técnicos com a equipe de pesquisa (WOODS et al., 2019).

O usuário precisa sentir-se seguro e confortável por meio da ajuda disponibilizada diante das dicas e esclarecimentos, buscando favorecer a compreensão, inclusive para aqueles que já têm acesso à tecnologia (CURRIE; PHILIP; ROBERTS, 2015; HEFFERNAN et al., 2016; ISAKOVIĆ et al., 2016). A motivação e o envolvimento no tratamento é algo intrínseco de cada participante. Além disso, pode depender de vários fatores e sofrer alterações no decorrer do tempo (KUJALA et al., 2011), merecendo atenção tanto do paciente quanto dos profissionais envolvidos (ALANZI; ISTEPANIAN; PHILIP, 2016). Na questão 8 identificou-se a necessidade de dedicar mais tempo para acompanhar a evolução da experiência, a fim de averiguar os fatores que podem tornar o aplicativo mais atrativo para os usuários. No trabalho de (PARKER, 2019), o SUPR-Qm também foi utilizado para avaliação da usabilidade e experiência de um aplicativo, no qual os autores declaram que uma compreensão básica dos recursos, funcionalidades e design é fundamental para o sucesso e para a ampla adoção de qualquer aplicativo.

Quanto aos resultados do TAM, obteve-se maior concordância na questão que buscou identificar se o aplicativo foi importante para o cuidado com a saúde (questão 3). O aplicativo auxiliou no cuidado direcionado a pressão arterial e estimulou o registro dos valores, em conformidade com os aplicativos de saúde na atenção destinada à hipertensão11. Além disso, os relatos dos pacientes demonstraram que o aplicativo despertou o interesse do autocuidado, de saber como proceder durante o período de utilização, aumentando o monitoramento da saúde. Quanto as exigências mentais, na questão 8, os usuários alegaram que o aplicativo exigiu empenho para aprender a utilizar, aumentando a concentração de cada participante (CHANDRA; OAKLEY; SILVA, 2012; SARKAR et al., 2016). Para utilizar o aplicativo foram oferecidos aos usuários treinamentos, orientações, momentos para esclarecimento de dúvidas, e demonstrações de utilização (questão 12). Alguns tiveram dificuldades para compreender a questão 7 , como no estudo de (STÜTZ et al., 2017), no qual os contratempos iniciais foram amenizados com as instruções disponibilizadas.

No quesito do que seria melhor, se o uso do aplicativo ou manter o método de acompanhamento habitual, alguns preferiram manter o contato usual, com visitas periódicas aos profissionais (questão 13), equivalente as contribuições de (DOU et al., 2017). Para a maioria dos participantes do estudo atual o aplicativo foi uma novidade, o que acarretou em vários questionamentos iniciais (questão 14). Como no estudo de (STÜTZ et al., 2017), a minoria tinha o hábito de usar o aplicativo no cuidado com a saúde. As principais dúvidas apontadas pelos participantes foram sobre $\mathrm{o}$ aplicativo, suas funcionalidades e segurança, assim como apontado por (SUN et al., 2017), em um estudo com pacientes chineses portadores de doenças crônicas. Essas preocupações podem ocorrer quando os usuários possuem a intenção de continuar utilizando o aplicativo, e, após os esclarecimentos, as inseguranças podem diminuir gradativamente (SUN et al., 2017). (VORRINK et al., 2017) acrescentam que os profissionais de saúde também têm dúvidas semelhantes como as descritas anteriormente.

Para análise da questão de pergunta aberta, "O que você mais gostou no aplicativo e por quê?", a maioria dos participantes afirmaram ter pouco conhecimento com aplicativos direcionados para a saúde, conforme pode ser observado na resposta "não tinha o costume de usar (P7)". Outro fator foi o tempo limitado de uso e o desinteresse inicial "consegui usar por pouco tempo (P2)"; "tive pouco interesse no início (P3)”.

Aspectos positivos mencionados pelos participantes são evidenciados pelas respostas "eu tinha o compromisso de verificar os sinais vitais (P8)"; "o aplicativo diz se está boa a pressão (P6)"; "poder controlar a pressão, corrigir hábitos alimentares e fazer exercícios (P10)"; "é mais fácil resolver os problemas (P11)". Para o P5, P1 e P9, a experiência e a importância do controle da pressão de forma regular foram os aspectos destacados: "foi muito bom conferir a pressão com frequência e foi uma experiência importante, ajuda muito (P5)"; "gostei por ficar sabendo e controlando "; "muito bom poder conferir a pressão (P1)". Uma das funcionalidades do aplicativo é a bonificação, desenvolvida para estimular e aumentar o interesse dos usuários em registrar os dados (CECHETTI et al., 2019). Esse aspecto foi considerado positivo pelos participantes, como pode ser observado pelo relato: "gosto quando ganho pontos, isto é 
gratificante (P4)". As características mencionadas pelos participantes associam-se com as tendências para a diminuição da pressão arterial mediante hábitos saudáveis (ASHOORKHANI et al., 2016).

Alguns participantes relataram problemas relacionados a funcionalidades e redução da frequência de uso, também presentes nos achados de (VORRINK et al., 2017). Outros participantes demonstraram pouca confiança em registrar os dados no aplicativo, como identificaram (SARKAR et al., 2016) e (DOU et al., 2017). Isso pode ter sido ocasionado por assuntos pessoais de cada participante, e pela limitação de alguns dispositivos móveis, como pode ser observado na resposta: "só que teria que ter um aparelho melhor (P5)".

Os resultados demonstraram que, frequentemente, adultos mais jovens tendem a ser mais positivos e se adaptam à tecnologia com mais facilidade, de acordo com (SUN et al., 2017) e (PARK et al., 2017). Ainda, outros autores afirmam que essas tendências podem ocorrer quando há diferentes faixas etárias em um mesmo estudo (DAVIS et al., 2015; SUN et al., 2017).

Entre as limitações do estudo cita-se o fato de ser um estudo piloto com tamanho amostral reduzido. Porém, o estudo é promissor, pois são poucos os resultados do uso de tecnologias no tratamento e monitoramento de pacientes com hipertensão.

\section{CONCLUSÃO}

$\mathrm{O}$ estudo identificou a necessidade e o interesse que os pacientes têm em utilizar a tecnologia para o acompanhamento e o controle da pressão arterial. Nota-se que, com o passar dos anos, a tecnologia é inserida de forma gradual servindo de apoio nos cuidados das pessoas e dos profissionais. Inicialmente pode haver resistência quanto a adaptação, falta de confiança, e frustações com o aplicativo. Porém, gradativamente, os usuários identificam benefícios e demonstram interesse em continuar utilizando a tecnologia na autogestão de saúde.

As tecnologias móveis podem de forma alternativa vir a contribuir para o autogerenciamento da doença crônica por parte do paciente, familiar, e equipe de saúde, por ser uma ferramenta simples e de fácil acesso para a maioria da população. Entretanto, as soluções precisam ser melhoradas para que os usuários divulguem as recomendações e o uso para seus familiares, colegas e amigos como ferramenta de monitoramento e gerenciamento das condições de saúde.

Destaca-se ser fundamental identificar as experiências e opiniões para que o processo de aceitação seja aprimorado. Dessa forma, sugere-se a realização de estudos futuros para analisar a experiência cumulativa ao longo do tempo, buscando-se comparar os resultados antes e depois da interação com o aplicativo. Este estudo piloto possibilitou o teste de viabilidade dos instrumentos que foram empregados no método do ensaio clínico de (VOLPI et al., 2021).

\section{REFERÊNCIAS}

ALANZI, T.; ISTEPANIAN, R.; PHILIP, N. Design and Usability Evaluation of Social Mobile Diabetes Management System in the Gulf Region. JMIR Research Protocols, v. 5, n. 3, p. e93, 2016.

ANDERSEN, T. O. et al. Understanding patient experience. Proceedings of the 11th EAI International Conference on Pervasive Computing Technologies for Healthcare. Anais...New York, NY, USA: ACM, 2017

ASHOORKHANI, M. et al. Comparing the effectiveness of the BPMAP (Blood Pressure Management Application) and usual care in self-management of primary hypertension and adherence to treatment in patients aged 30-60 years: study protocol for a randomized controlled trial. Trials, v. 17, n. 1, p. 511, 2016.

BIDUSKI, D. et al. Assessing long-term user experience on a mobile health application through an in-app embedded conversation-based questionnaire. Computers in Human Behavior, v. 104, p. 106169, mar. 2020.

BOUDREAUX, E. D. et al. Evaluating and selecting mobile health apps: strategies for healthcare providers and healthcare organizations. Translational Behavioral Medicine, v. 4, n. 4, p. 363-371, 2014.

CAVALCANTE, E.; RIVERO, L.; CONTE, T. MAX: A Method for Evaluating the Post-use User eXperience through Cards and a Board. jul. 2015 


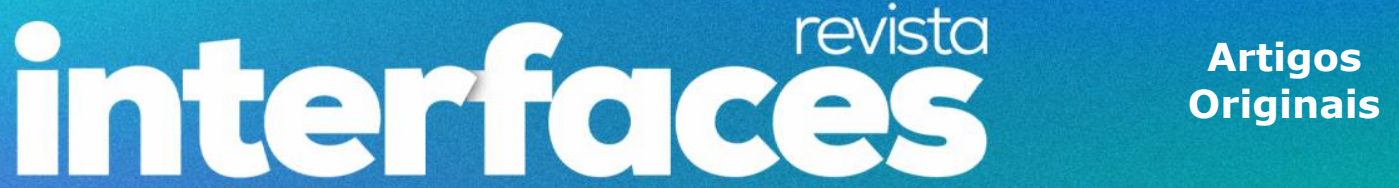

\section{V.10 N.1 (2022) ISSN: $2317-434 X$}

CECHETTI, N. P. et al. Developing and implementing a gamification method to improve user engagement: A case study with an m-Health application for hypertension monitoring. Telematics and Informatics, v. 41, p. 126138, ago. 2019.

CHANDRA, H.; OAKLEY, I.; SILVA, H. User needs in the performance of prescribed home exercise therapy. Proceedings of the 2012 ACM annual conference extended abstracts on Human Factors in Computing Systems Extended Abstracts - CHI EA '12. Anais...New York, New York, USA: ACM Press, 2012

CURRIE, M.; PHILIP, L. J.; ROBERTS, A. Attitudes towards the use and acceptance of eHealth technologies: a case study of older adults living with chronic pain and implications for rural healthcare. BMC Health Services Research, v. 15, n. 1, p. 162, 2015.

DAVIS, F. D.; BAGOZZI, R. P.; WARSHAW, P. R. User Acceptance of Computer Technology: A Comparison of Two Theoretical Models. Management Science, v. 35, n. 8, p. 982-1003, 1989.

DAVIS, R. et al. Theories of behaviour and behaviour change across the social and behavioural sciences: a scoping review. Health Psychology Review, v. 9, n. 3, p. 323-344, 2015.

DE MARCHI, A. C. B. et al. An Electronic Health Platform for Monitoring Health Conditions of Patients With Hypertension in the Brazilian Public Health System: Protocol for a Nonrandomized Controlled Trial. JMIR Research Protocols, v. 9, n. 1, p. e15299, 20 jan. 2020.

DOU, K. et al. Patients' Acceptance of Smartphone Health Technology for Chronic Disease Management: A Theoretical Model and Empirical Test. JMIR mHealth and uHealth, v. 5, n. 12, p. e177, 2017.

FREITAS, P. DA S. et al. Uso de serviços de saúde e de medicamentos por portadores de Hipertensão e Diabetes no Município do Rio de Janeiro, Brasil. Ciência \& Saúde Coletiva, v. 23, n. 7, p. 2383-2392, 2018.

HARICHARAN, H. J. et al. Health promotion via SMS improves hypertension knowledge for deaf South Africans. BMC Public Health, v. 17, n. 1, p. 663, 2017.

HEFFERNAN, K. J. et al. Guidelines and Recommendations for Developing Interactive eHealth
Apps for Complex Messaging in Health Promotion. JMIR mHealth and uHealth, v. 4, n. 1, p. e14, 2016.

ISAKOVIĆ, M. et al. Usability Pitfalls of Diabetes mHealth Apps for the Elderly. Journal of Diabetes Research, v. 2016, p. 1-9, 2016.

JOHNSON, R. B.; ONWUEGBUZIE, A. J.; TURNER, L. A. Toward a Definition of Mixed Methods Research. Journal of Mixed Methods Research, v. 1, n. 2, p. 112 133, 2007.

KITT, J. et al. New Approaches in Hypertension Management: a Review of Current and Developing Technologies and Their Potential Impact on Hypertension Care. Current Hypertension Reports, v. 21, n. 6, p. 44, 2019.

KUJALA, S. et al. UX Curve: A method for evaluating long-term user experience. Interacting with Computers, v. 23, n. 5, p. 473-483, 2011.

LU, C. et al. The Use of Mobile Health Applications to Improve Patient Experience: Cross-Sectional Study in Chinese Public Hospitals. JMIR mHealth and uHealth, v. 6, n. 5, p. e126, 2018.

MÁRQUEZ CONTRERAS, E. et al. Specific hypertension smartphone application to improve medication adherence in hypertension: a clusterrandomized trial. Current Medical Research and Opinion, v. 35, n. 1, p. 167-173, 2019.

PARK, D. Y. et al. Implications for Training on Smartphone Medication Reminder App Use by Adults With Chronic Conditions: Pilot Study Applying the Technology Acceptance Model. JMIR Formative Research, v. 1, n. 1, p. e5, 2017.

PARKER, K. Competition and athlete engagement to improve nutrition: a usability study of the mobile application BSUathleats. [s.1.] Ball State University, 2019.

SARKAR, U. et al. Usability of Commercially Available Mobile Applications for Diverse Patients. Journal of General Internal Medicine, v. 31, n. 12, p. 1417-1426, 2016.

SAURO, J.; ZAROLIA, P. SUPR-Qm: A Questionnaire to Measure the Mobile App User Experience. J. Usability Studies, v. 13, n. 1, p. 17-37, 2017. 
SILVA, T. DE CARVALHO DA et al. Método de adesão ao tratamento de pacientes hipertensos. ARCHIVES OF

HEALTH INVESTIGATION, v. 6, n. 4, 2017.

STÜTZ, T. et al. Mobile Phone-Supported Physiotherapy for Frozen Shoulder: Feasibility Assessment Based on a Usability Study. JMIR Rehabilitation and Assistive Technologies, v. 4, n. 2, p. e6, 2017.

SUN, L. et al. Facilitators and barriers to using physical activity smartphone apps among Chinese patients with chronic diseases. BMC Medical Informatics and Decision Making, v. 17, n. 1, p. 44, 2017.

TORO-RAMOS, T. et al. Efficacy of a mobile hypertension prevention delivery platform with human coaching. Journal of Human Hypertension, v. 31, n. 12, p. 795-800, 2017.

VO, V.; AUROY, L.; SARRADON-ECK, A. Patients' Perceptions of mHealth Apps: Meta-Ethnographic Review of Qualitative Studies. JMIR mHealth and uHealth, v. 7, n. 7, p. e13817, 2019.

VOLPI, S. S. et al. Using a mobile health app to improve patients' adherence to hypertension treatment: a nonrandomized clinical trial. PeerJ, v. 9, p. e11491, 28 maio 2021.

VORRINK, S. et al. Perceptions of Patients With Chronic Obstructive Pulmonary Disease and Their Physiotherapists Regarding the Use of an eHealth Intervention. JMIR Human Factors, v. 4, n. 3, p. e20, 2017.

WOODS, L. S. et al. Patients' Experiences of Using a Consumer mHealth App for Self-Management of Heart Failure: Mixed-Methods Study. JMIR Human Factors, v. 6, n. 2, p. e13009, 2019. 